\title{
A hierarchical layout design method based on rubber band potential- energy descending
}

\author{
Cheng Yi Ou ${ }^{1}$, Hai Hua Xiao ${ }^{1}$, Jun Yan $\mathrm{Ma}^{1, \mathrm{a}}$ and Xiao Ping Liao ${ }^{2}$ \\ ${ }^{1}$ Mechancal manufacturing \& automation, college of mechanical \& engineering, Guangxi University, Nanning, Guangxi, China \\ ${ }^{2}$ Mechancal manufacturing \& automation, science and technology department, Guangxi University, Nanning, Guangxi, China
}

\begin{abstract}
Strip packing problems is one important sub-problem of the Cutting stock problems. Its application domains include sheet metal, ship making, wood, furniture, garment, shoes and glass. In this paper, a hierarchical layout design method based on rubber band potential-energy descending was proposed. The basic concept of the rubber band enclosing model was described in detail. We divided the layout process into three different stages: initial layout stage, rubber band enclosing stage and local adjustment stage. In different stages, the most efficient strategies were employed for further improving the layout solution. Computational results show that the proposed method performed better than the GLSHA algorithm for three out of nine instances in utilization.
\end{abstract}

\section{Introduction}

Strip packing problems(SPP) is one of the Cutting stock problems(CSP) which presented by R.C. Art[1] early in 1996. SPP aimed at maximizing the utilization of materials with optimal stock layout design. Because of its potential application and theoretical research value, a large number of researchers are devoted to this field from different industry, such as sheet metal, ship making, wood, furniture, garment, shoes and glass.

According to the latest classification rules from Wäscher[2], SPP is defined as an open-dimensional optimal problem, in which the given strip material has fixed width but its length is infinite. So the target of SPP is finding the most compact layout solution with minimal length for a set of layout objects.

Algorithms for solving SPP can be classified into three categories: deterministic algorithms, indeterministic algorithms and algorithms in between. Deterministic algorithms, in general, would reach an deterministic layout solution on a low time consumption, such as mathematical programming[3]. However, solutions from deterministic algorithm are relatively optimal. It can't meet actual application need. On the other hand, indeterministic algorithms own strong capability of global search, such as genetic algorithm(GA)[4], particle swarm algorithm(PSO)[5] and neural network algorithm[6]. But these algorithms usually consume a large amount of calculation time in their iteration operation. So algorithms between deterministic algorithms and indeterministic algorithms are a balance of both. They try to balance the relationship between time consumption and final optimal solution through combining specific heuristic rules and other intelligence algorithms, such as bottom-left placement algorithm(BL)[7].

However, the popular algorithms at present are still time consuming. In this paper, we introduce a hierarchical layout design method based on potentialenergy descending of rubber band. The inspiration of this method is from physical phenomena. When a rubber band enclosed several objects, these objects will move close to each other until forming a compact layout solution. The proposed hierarchical conception is to divide layout process into three stages for employing efficient strategies.

The remainder of the paper is organized as follows. Section 2 describes the SPP optimization model for irregular polygons. Section 3 introduces the rubber band enclosing model and describes some essential analysis methods. Section 4 describes the proposed hierarchical layout design model in detail. Section 5 presents the computer test results and compares the proposed method with others. Section 6 is summary and prospect of this paper.

\section{SPP optimization model}

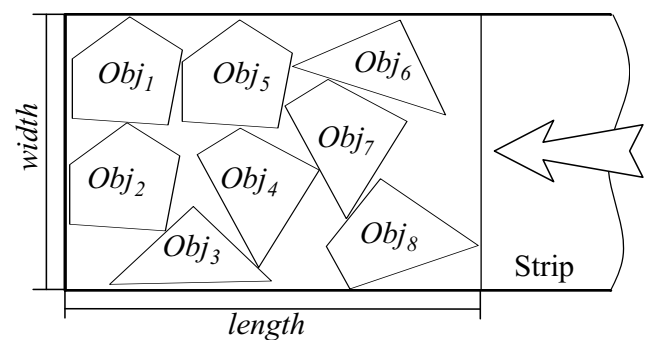

Figure1. The diagram of strip packing problems.

\footnotetext{
a Corresponding author: ouyi3427@126.com.
} 
As shown in Fig.1, given a set of layout objects $O \mathrm{Obj}_{1}, \mathrm{Obj}_{2} \ldots O \mathrm{Obj}_{n}$ and a strip $\mathrm{Str}$ with specific width $w$, find a group of proper positions and angles $p_{1}\left(x_{1}, y_{1}, a n g_{1}\right) \ldots p_{n}\left(x_{n}, y_{n}, a n g_{n}\right)$ for every objects so as to place them into the strip minimizing the length of utilized strip without any overlap. It can be expressed by the optimization model shown below.

$$
\begin{aligned}
& \text { Find } \quad p_{i}\left(x_{i}, y_{i}, \text { ang }_{i}\right) \\
& \text { Min } \quad l \\
& \text { S.T. } \quad \mathrm{Obj}_{i} \subseteq S t r \\
& \quad \operatorname{Obj}_{i} \cap O b j_{j}=\phi(i, j=1,2 . . n)
\end{aligned}
$$

In other words, finding the minimal $l$ is equivalent to maximize the material utilization of the rectangular region used. The utilization $u$ can be expressed by equation (2).

$$
u=\frac{\sum \operatorname{Area}\left(\mathrm{Obj}_{i}\right)}{w \times l} \times 100 \%
$$

Moreover, we only concentrate on irregular polygon layout problems because that any $2 \mathrm{D}$ objects can be instead of polygon approximately.

\section{Rubber band enclosing model}

Assume that several objects are enclosed by a stretched rubber band shown in Fig.2. Under the impact of elastic force from rubber band, all objects will move to each other as close as possible forming a compact layout in the end. In addition, potential energy of rubber band reaches to minimum. This layout process is the proposed rubber band potential-energy descending model(RPD).

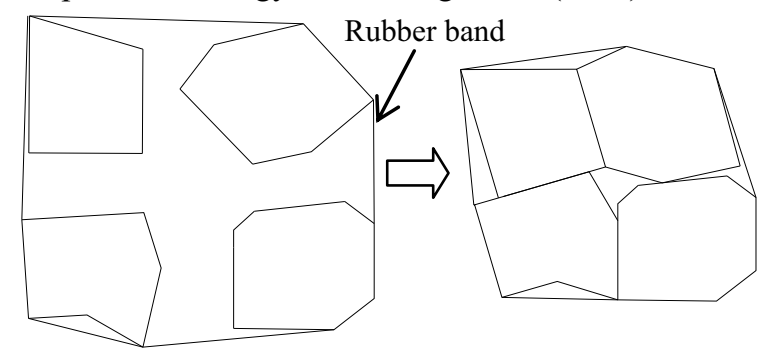

Figure 2. Rubber band enclosing model.

Some problems must be settled before constructing RPD model:

1. The construction of rubber band.

2. Force analysis and motion analysis for objects.

3. Convergence condition.

\subsection{Construction of rubber band}

The problem of rubber band's construction is equivalent to construct the convex hull of the vertices set of all polygons. Here we utilize Graham algorithm[8] to produce the convex hull. Graham algorithm is a classical convex hull algorithm which performs effectively when the scale of the given point set is small.

\subsection{Force analysis}

An object would get forces from rubber band or other objects. Force from rubber band is called elastic force denoted by $\vec{F}_{R_{i}}$, and force from other objects is called collision force denoted by $\vec{F}_{O b j}$.

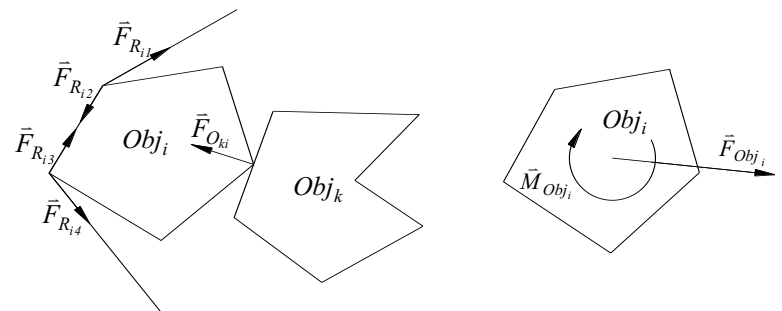

Figure 3. Force analysis.

Figure 4. Resultant force and moment.

Firstly, as shown in Fig.3, calculate all elastic forces $\vec{F}_{R_{i 1}}, \vec{F}_{R_{i 2}} \ldots \vec{F}_{R_{i n}}$. Then utilize ray scan method[9] to check which object is under collision. Calculate all collision forces $\vec{F}_{O_{i 1}}, \vec{F}_{O_{i 2}} \ldots \vec{F}_{O_{i n}}$.

Secondly, calculate the resultant force with equation (3) shown as follow.

$$
\vec{F}_{O b j_{i}}^{\prime}=\sum \vec{F}_{R_{i n}}+\sum \vec{F}_{O_{i n}}
$$

Finally, replace $\vec{F}_{O b j_{i}}^{\prime}$ with a force $\vec{F}_{O b j_{i}}$ through the centroid and a moment $\vec{M}_{O b j_{i}}$ which can be calculate with the follow equation. Fig.4 shows the consequence of force analysis.

$$
\vec{M}_{O b j_{i}}=\vec{F}_{O b j_{i}} \cdot d
$$

\subsection{Motion analysis}

The continuous layout process is transformed into discrete time $t\left(t_{1}, t_{2} . . t_{\infty}\right)$ for motion analysis. An assumption has been made in which the force and moment applied to objects are invariable in each time step. That is to say objects only do uniformly accelerated motion. Then let $P_{O b j_{i}}^{(k)}\left(x_{i}, y_{i}, a_{i n g}\right)$ denotes present displacement vector of i-th object in k-th time step. $\vec{V}_{O b j_{i}}^{(k)}\left(v x_{i}, v y_{i}, v \omega_{i}\right)$ denotes present velocity vector. $\vec{a}_{O b j_{i}}^{(k)}\left(\alpha_{i}, \beta_{i}, \gamma_{i}\right)$ denotes present acceleration vector. According to the Newton's laws, $P_{O b j_{i}}^{(k)}, \vec{V}_{O b j_{i}}^{(k)}$ and $\vec{a}_{O b j_{i}}^{(k)}$ can be easily calculated by the following equation. Here we use area $A_{O b j_{i}}$ instead of object's mass and $J_{O b j_{i}}$ denotes the moment of inertia .

$$
\begin{aligned}
& \vec{a}_{O b j_{i}}^{(k)} \cdot\left(\alpha_{i}, \beta_{i}\right)=\frac{\vec{F}_{O b j_{i}}^{(k)}}{A_{O b j_{i}}} \quad \vec{a}_{O b j_{i}}^{(k)} \cdot \gamma_{i}=\frac{\vec{M}_{O b j_{i}}^{(k)}}{J_{O b j_{i}}} \\
& \vec{V}_{O b j_{i}}^{(k)}=\vec{V}_{O b j_{i}}^{(k-1)}+\vec{a}_{O b j_{i}}^{(k)} \cdot t^{(k)} \\
& \vec{P}_{O b j_{i}}^{(k)}=\vec{V}_{O b j_{i}}^{(k-1)} \cdot t^{(k)}+\frac{1}{2} \vec{a}_{O b j_{i}}^{(k)} \cdot\left(t^{(k)}\right)^{2}
\end{aligned}
$$

The new solution can be calculated by the following equation.

$$
S^{(k)}=S^{(k-1)}+\left\lfloor\vec{P}_{O b j_{1}}^{(k)}, \vec{P}_{O b j_{2}}^{(k)} \ldots \vec{P}_{O b j_{n}}^{(k)}\right\rfloor
$$




\subsection{Convergence condition}

Potential-energy of rubber band denoted by $e$ is descending all the time until layout process get the local minimal. When the rate of change $\Delta e$ reduces very close to zero, we will consider the minimal has been found. And $\Delta e$ can be calculated by equation (7).

$$
\begin{aligned}
& e^{(k)}=\frac{1}{2} K \cdot\left(\Delta x^{(k)}\right)^{2} \\
& \Delta e=\left|e^{(k)}-e^{(k-1)}\right| \leq \varepsilon
\end{aligned}
$$

$K$ denotes stiffness of rubber band and $\Delta x$ denotes shape variable. $\varepsilon$ is a very small positive number.

\section{Hierarchical layout design model}

Most of popular hybrid algorithms place objects following a certain sequence one by one until all objects are placed in. And the duty of intelligence algorithm is to produce more potential sequence by iteration. These algorithms are time consuming. Actually, the layout process can be divided into different stages. A better choice is to employ the most suitable strategy in corresponding stages. That is the hierarchical layout design method(HLD).

In this paper we divide layout process into three stages: initial layout stage, rubber band enclosing stage and local adjustment stage. The flow chart of HLD method has been shown in Fig.6.

\subsection{Initial layout stage}

In HLD, rubber band enclosing contributes a lot to layout solution. Initial layout position of each object is the key to rubber band enclosing model. Different initial layout will lead to variable solutions as shown in Fig.5. So the target in initial layout stage is to find a relatively compact solution for further layout design. A rectangle placement strategy based on exhaustive search is proposed here.

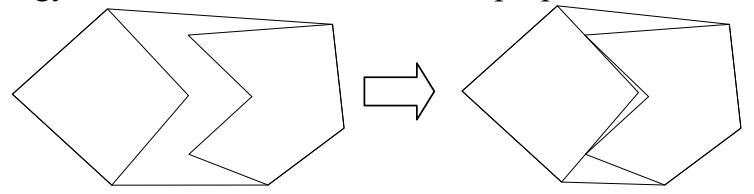

(a) Initial layout 1

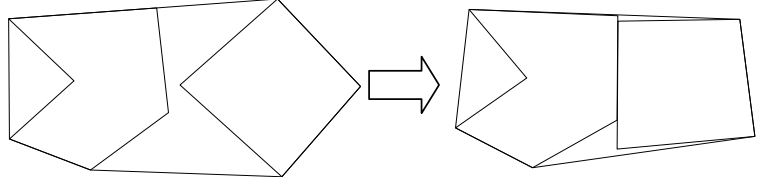

(b) Initial layout 2.

Figure 5. Different initial layouts lead to different consequence.

\subsubsection{Minimum rectangle bounding box}

Here a minimum rectangle bounding box is utilized to replace polygons for initial layout. The following describes the basic procedure for the construction of minimum rectangle.

(1)Produce the convex hull of polygon.
(2)Rotate convex hull in a counter clockwise direction until one edge parallels to the horizontal direction.

(3)Find $x_{\text {min }}, x_{\text {max }}, y_{\text {min }}, y_{\text {max }}$ from all vertices of convex hull. Use the value $x_{\max }-x_{\min }$ as length and $y_{\max }-y_{\min }$ as width of rectangle.

(4)Record the present rotation angle and area of rectangle.

(5)Repeat the above four steps until every edge is calculated. Then choose the rectangle with minimum area as minimum rectangle bounding box.

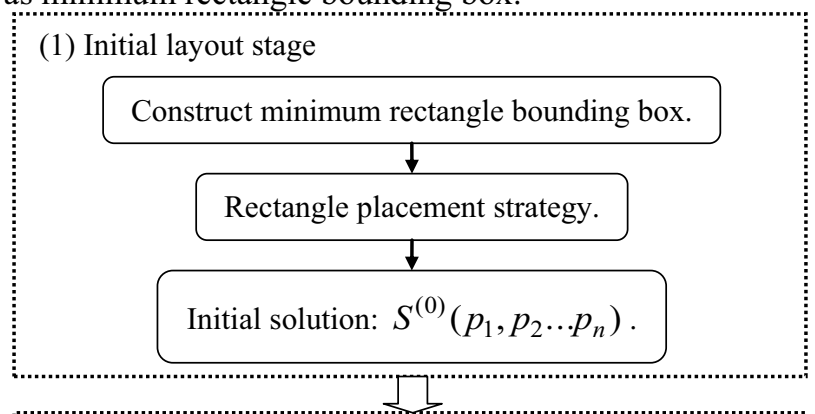

(2) Rubber band enclosing stage

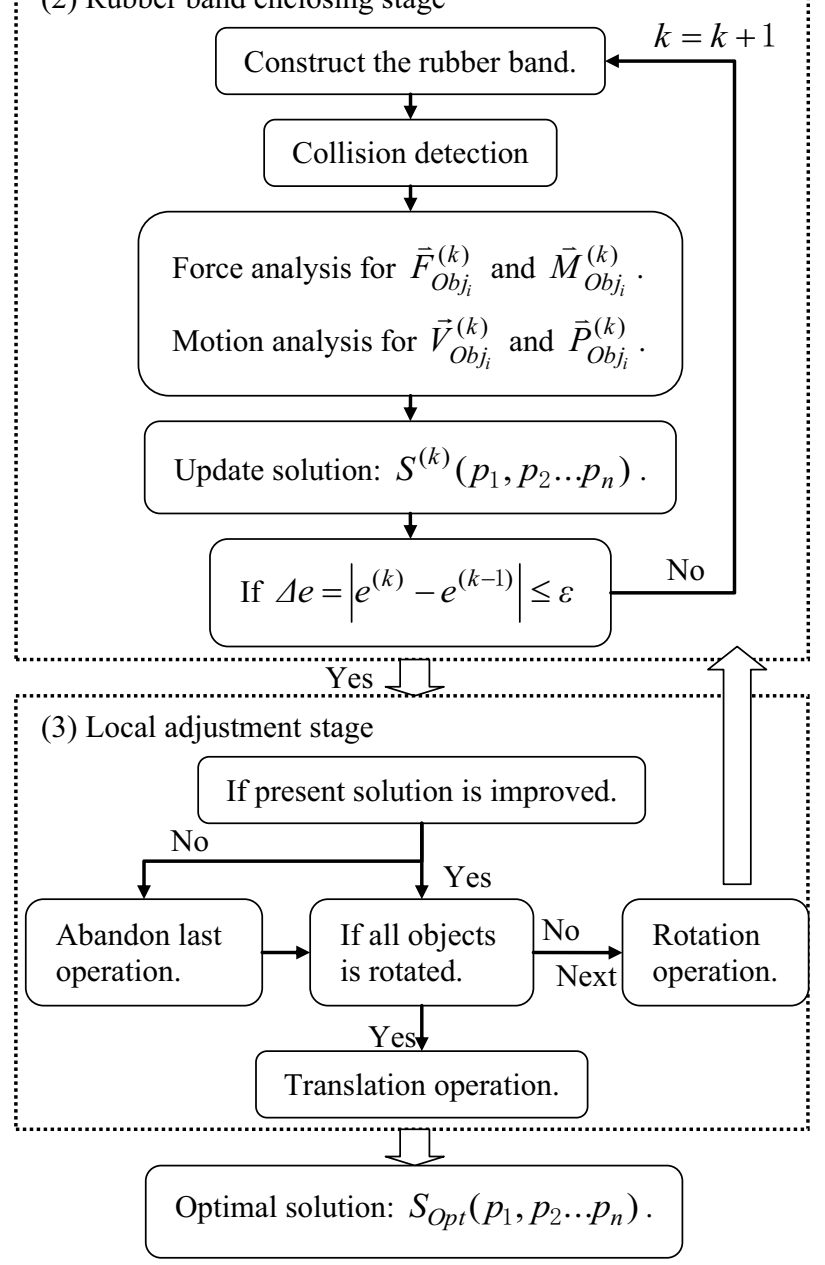

Figure 6. The flow chart of HLD method.

\subsubsection{Rectangle placement strategy}

Classical algorithms for rectangle packing include BL algorithm and lowest horizontal search algorithm[10]. These algorithms usually spend much computation time on their iteration. However, a relatively optimal solution 
not the most is expected to be found on a reasonable time. So iteration is unnecessary. The rectangle placement strategy is described in the following.

(1)Sort all rectangle bounding boxes from large to small according to the area size.

(2)Implement lowest horizontal search for each rectangle and their $90^{\circ}$ rotation version.

(3) Compare the utilization after one rectangle is packed in.

(4)Choose the case in which the highest utilization is got from all cases.

(5)Remove the packed rectangle from the list and return to step (2).

As shown in Fig.7, rectangle is allowed to rotate $90^{\circ}$ and each time the case with highest utilization would be chosen for next placement operation. Obviously, the proposed strategy needn not optimize its packing sequence by time-consuming iteration.

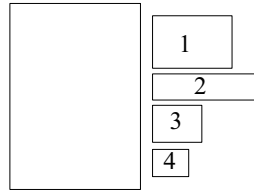

(a)

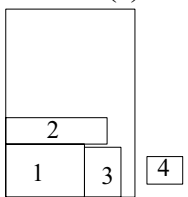

(d)

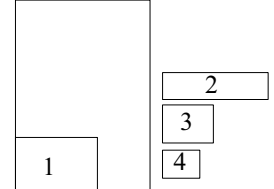

(b)

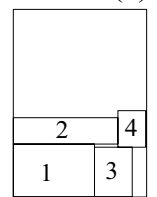

(e)

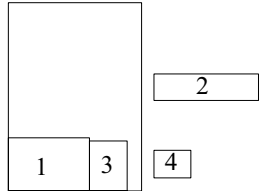

(c)
Figure 7. Diagram of rectangle placement strategy.

\subsection{Rubber band enclosing stage}

An initial solution $S^{(0)}\left(p_{1}, p_{2} \ldots p_{n}\right)$ has been acquired after initial layout. The rubber band enclosing model will be employed here to improve the present layout. We assume that the rubber band is fixed to the bottom of the given strip so that it can drag all objects to the direction leading to a minimum length of strip. This procedure is described in the following.

(1)Set $k=0$, stiffness $K$ and convergence precision $\varepsilon$.

(2)Construct the rubber band utilizing Graham algorithm and calculate the potential-energy $e^{(0)}$.

(3)Implement the ray scan method for collision detection.

(4)FOR $i=1$ TO $n$ DO

BEGIN

Calculate the elastic force $\vec{F}_{R_{i n}}^{(k)}$ and collision force $\vec{F}_{O_{\text {in }}}^{(k)}$.

Calculate the resultant force $\vec{F}_{O b j_{i}}^{(k)}$ and moment $\vec{M}_{O b j_{i}}^{(k)}$.

Calculate the velocity vector $\vec{V}_{O b j_{i}}^{(k)}$ and displacement vector $P_{O b j_{i}}^{(k)}$ at present time step $t^{(k)}$.

END

(5)Update the present solution $S^{(k)}\left(p_{1}, p_{2} \ldots p_{n}\right)$ with $\left\lfloor P_{O b j_{1}}^{(k)}, P_{O b j_{2}}^{(k)} \ldots P_{O b j_{n}}^{(k)}\right\rfloor$. Then construct the rubber band and calculate the potential-energy $e^{(k)}$.

(6)if $\Delta e=\left|e^{(k)}-e^{(k-1)}\right| \leq \varepsilon$, let $k=k+1$ and return to step(3). Otherwise output the optimal solution.

\subsection{Local adjustment stage}

Through the RPD operation, the solution is still relatively optimal and some operations are essential to improve it. Many factors can become obstacles to RPD's operation. The most common reason is that several objects get obstructed preventing themselves from moving close to each other further more. As a result, RPD would terminate in advance with an undesirable solution.

Here we employ rotation and translation strategy to adjust the RPD process so as to make rubber band can surround all objects more compactly.

\subsubsection{Compactness of different districts}

As shown in Fig.8, the rectangular region used of the strip is divided into four districts $D_{1}, D_{2}, D_{3}, D_{4}$ just like Cartesian coordinate is built at the centroid of this region. Assume that use object's area to denote its mass. So if the utilization reaches to $100 \%$, the centroid and the center of mass of all objects are on the same position. Thus, through checking the relative position of the two points, the district with low compactness can be easily distinguished.

Let point $\left(x_{m}, y_{m}\right)$ denotes center of mass of all objects, point $\left(x_{c}, y_{c}\right)$ denotes the centroid of the rectangle region, point $\left(x_{i}, y_{i}\right)$ denotes center of mass of $\mathrm{Obj}_{i}$. So the relative position vector can be calculated according to the following equation.

$$
\left(x_{r}, y_{r}\right)=\left(x_{m}, y_{m}\right)-\left(x_{c}, y_{c}\right)
$$

Point $\left(x_{m}, y_{m}\right)$ can be calculated by equation (9).

$\left(x_{m}, y_{m}\right)=\left(\sum \frac{\operatorname{Area}\left(\mathrm{Obj}_{i}\right)}{\sum \operatorname{Area}\left(\mathrm{Obj}_{n}\right)} \cdot x_{i}, \sum \frac{\operatorname{Area}\left(\mathrm{Obj}_{i}\right)}{\sum \operatorname{Area}\left(\mathrm{Obj}_{n}\right)} \cdot y_{i}\right)$

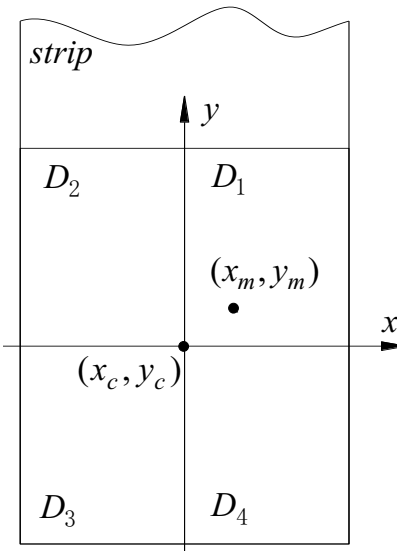

Figure 8. Diagram of the four districts.

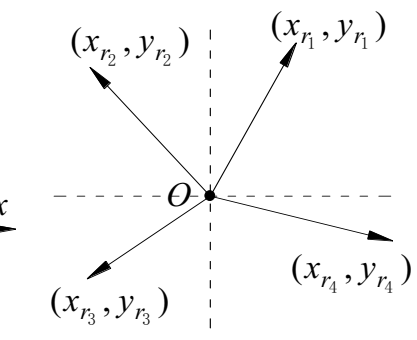

Figure 9. Relative position vector. 
If $\left(x_{r}, y_{r}\right) \in D_{1}$ shown in Fig.9, it reveals that its opposite region $D_{3}$ owns a low compactness. That means obstruction would most possibly occur in this district. And if $\left(x_{r}, y_{r}\right) \in D_{2}, D_{4}$ owns a low compactness. Similarly, it also goes for both cases $\left(x_{r}, y_{r}\right) \in D_{3}$ and $\left(x_{r}, y_{r}\right) \in D_{4}$.

\subsubsection{Rotation and translation strategy}

The target of rotation and translation strategy employed in this stage is to eliminate the influence of object's obstruction. Through rotation and translation operations, make the modulus of relative position vector reduce gradually to zero.

Firstly, select out all objects which is located in the district got by calculating relative position vector. Rotate them on a certain angle one by one. Then execute the rubber band enclosing operation after one object is rotated. Check whether the new solution owns a higher utilization than before. If so, accept the new solution and calculate the relative position vector again. Otherwise, give up the rotation operation to the present object and rotate next one. If all rotation operations applied to objects made no improvement, try to translate little objects artificially from other districts to fill the space non-utilized in this district. Translation operation must obey the basic principle that each operation would help to make the relative position vector go to $(0,0)$.

\section{Experiments}

HLD method was implemented in $\mathrm{C}++$ on a $\mathrm{PC}$ with an i3 dual-core $2.53 \mathrm{GHz}$ CPU and $2 \mathrm{~GB}$ of RAM. And a few famous benchmark instances from the ESICUP website were used to test the performance of our layout method (http://www.apdio.pt/esicup). These instances were listed in table 1 . The computational results were compared with the GLSHA algorithm and the SAHA algorithm[11].

In table 1, the first four columns summaries the basic information of these instances. But it's worth noting that all objects is allowed to rotate in any angle which is different from the original instances. The last three columns present the computational results of the proposed method and other two algorithms.
Comparing the computational results of 9 benchmark instances with other two algorithms, the proposed method obtained a better result than GLSHA algorithm for three instances out of 9 instances in the utilization. And each result was very close to the one come from SAHA algorithm. The appendix presents the optimal placements obtained by HLD method for these instances.

\section{Conclusions}

In this paper, a hierarchical layout design method was proposed. This HLD method is used for solving the strip packing problems. The layout process of HLD method is based on rubber band potential-energy descending. It includes three stages and corresponding strategies are employed to improve the optimal solution gradually. 9 benchmark instances were used for testing the performance of the proposed method. And the results show that the HLD method is quite promising with high utilization compared with other algorithms.

\section{Acknowledgments}

We sincerely acknowledge the support of National Nature Science Foundation of China (NO. 51265002).

\section{References}

1. R.C. Art. IBM Cambridge Scientific Centre (1966).

2. G. Wäsche, H. Haußner, H. Schumann. EUR J OPER RES, 183, 1109-1130 (2007).

3. J.E. Beasley. Operations Research, 33, 49-64 (1985).

4. J.H. Holland. De Luca, Control \& Artificial Intelligence University of Michigan (1975).

5. K. James, R. Eberhart. IEEE, 4, 1942-1948 (1995).

6. C. Wee Liew. IEEE, 12, 67-73 (1997).

7. S. Jakobs. EJOR, 88, 165-181 (1996).

8. R.L. Graham. Inf. Proc. Lett, 72, 132-133 (1972).

9. L. Xiangsha, L. Fengying, M. Junyan, L. Xiaoping. Machinery Design \& Manufacture. 185-188 (2015).

10. W. Zhuting, L. Lin, C. Hao, L. Xinbao. J ENG DESIGN, 16, 98-102 (2009).

11. A.M. Gomes, J.F. Oliveira. EJOR, 171, 811-829 (2006).

\begin{tabular}{lcccccc}
\multicolumn{7}{c}{ Table 1. Benchmark instances and computational results. } \\
\hline Data sets & $\begin{array}{c}\text { number of } \\
\text { polygon types }\end{array}$ & $\begin{array}{c}\text { Total number } \\
\text { of polygons }\end{array}$ & Width of strip & GLSHA & SAHA & HLD \\
\hline Albano & 8 & 24 & 4900 & 0.8641 & 0.8742 & 0.8584 \\
Blaz1 & 7 & 28 & 15 & 0.8182 & 0.8359 & 0.7513 \\
Dagli & 10 & 30 & 60 & 0.8549 & 0.8714 & 0.86 .71 \\
Dighe1 & 16 & 16 & 100 & 0.8267 & 1 & 0.8463 \\
Dighe2 & 10 & 10 & 100 & 1 & 1 & 0.8248 \\
Fu & 12 & 12 & 38 & 0.8757 & 0.9095 & 0.8731 \\
Jakobs1 & 25 & 25 & 40 & 0.8166 & 0.7888 & 0.7780 \\
Jakobs2 & 25 & 25 & 70 & 0.7423 & 0.7728 & 0.7652 \\
Mao & 9 & 20 & 2550 & 0.8101 & 0.7999 & 0.7970 \\
\hline
\end{tabular}

